\title{
Development of transformable reflectors of mirror space antennas
}

\author{
Sergey Reznik ${ }^{1}$, Pavel Prosuntsov ${ }^{1}$, Oleg Denisov ${ }^{1}$, Liliana Denisova ${ }^{1}$, Vladimir Zavaryev ${ }^{2}$, \\ and Konstantin Mikhailovskiy, ${ }^{1, *}$ \\ ${ }^{1}$ Bauman Moscow State Technical University, 105005, 2-nd Baumanskaya st., 5, Moscow, Russia \\ ${ }^{2}$ OJSK «Firm «Triinvest», 115035, Sadovnicheskaya st., 35, Moscow, Russia
}

\begin{abstract}
Currently, the task of designing large structures of space antennas is becoming increasingly important due to active research and development in the area of creating remote sensing and communication satellites. Designing such structures is a complicated task due to geometric features, a large number of joints, compactness requirements, as well as operating conditions of the outer space. These factors require special methods for assessing the workability of these structures. This paper presents the results of simulation and experimental studies aimed at creating transformable antenna reflectors. The results of choosing general structural layouts are considered. It is noted that the main reason for the reflector shape distortion is non-uniform heating by the solar radiation and radiation fluxes coming from the Earth. The paper presents the results of numerical modeling of temperature and stress-strain state of transformable antenna reflectors from metallic mesh sheet meeting the requirements for operating on the geostationary orbit.
\end{abstract}

\section{Introduction}

Promising avenues for the development of communication systems and systems for remote sensing of the Earth include the creation of spacecrafts with transformable antenna reflectors. [1-4]. Antennas with large reflectors «AstroMesh» (rim structure) are used in space communication satellites «Thuraya», «MBCO», «Inmarsat», «GlobalStar». JSC Special Design Bureau of Moscow Power Engineering Institute (OKB MEI), which is a part of the JSC «Russian Space Systems», developed a large truss structure reflector for radar space antenna. JSC «NPO Lavochckin» and the Astro Space Center of the Lebedev Physical Institute of Russian Academy of Sciences developed a unique project of space radio telescope «Radioastron» with a petal-type load-bearing structure. This telescope had been working for 8.5 years on the near-Earth orbit since 2010. JSC «ISS - Reschetnev Company» is working in cooperation with several companies and the Astro Space Center of the Lebedev Physical Institute of Russian Academy of Sciences on creating a radio telescope «Millimetron» with a unique temperature control system of the composite load-bearing structure. This was made possible through the advent of polymer composite materials (PCM) with high specific strength and stiffness, polymer fiber webs, metallic woven mesh sheets from small diameter $(15-50 \mu \mathrm{m})$ gold-plated tungsten or molybdenum fibers.

* Corresponding author: konst mi@mail.ru 
A common problem of designing transformable antenna reflectors is to ensure the form and dimensional stability under varying thermal regimes causing temperature deformations. In these conditions, computer modeling is required for creating rational structural and technological solutions [5-10].

There is a need for the theoretical and technological foundation to be laid out for solving the problem of developing and manufacturing large transformable antenna reflectors with the aperture diameter of up to $100 \mathrm{~m}$ and a high-precision reflecting surface with long service life.

\section{Goal, problem statement and modeling method}

The paper aims to develop a method of choosing and justifying principal geometric models of super-light transformable antenna reflectors made using polymer composite materials and parametric modeling.

The distinctive feature of these reflectors is in using polymer composite materials, specifically CFRPs (carbon fiber reinforced polymers), for creating structural load-bearing elements in the form of hollow rods and reflecting surfaces from metallic mesh sheets.

During motion on the orbit and in the conditions of the outer space, large transformable antenna reflectors are exposed to a set of physical factors, which vary in both nature and intensity. The main factors are ultra-high vacuum, solar and ionizing radiation, bombardment by atomic and molecular particles, micrometeoroids and small space debris particles, thermal cycling (up to several thousand cycles per year in a wide temperature range, for example, from 80 to $450 \mathrm{~K}$ ).

The method is in modeling these factors with a multiphysics analysis; the method includes the following stages:

- Building geometric models of large transformable antenna reflectors meeting the requirements for the exterior construction, fixture and joining conditions, joints for enabling compact folding configuration;

- Building finite element models of design variants;

- Materials' selection and definition of physico-mechanical and thermal characteristics for the operating temperature range;

- Modeling temperature state to calculate temperature gradients in the elements of the transformable antenna reflector structure made from PCM being operated in the environment for a given orbit type;

- Modeling stress-strain state of the elements of the large transformable antenna reflector structure caused by the temperature gradients calculated at the previous stage;

- Choosing the design of the transformable antenna reflector from PCM having the minimum mass and meeting the requirements for shape and dimensional stability.

\section{Approbation of method and modeling results}

The subject of this study is a transformable antenna reflector for space communication systems. The reflector construction is comprised of a hub and a frame with longitudinal and transverse rods, as well as shape-forming rods maintaining the parabolic form of the reflector (Fig. 1). The longitudinal rods are fixed in the hub, and the hub has eight slots for axles around which the hinges of the foldable longitudinal rods can rotate. 


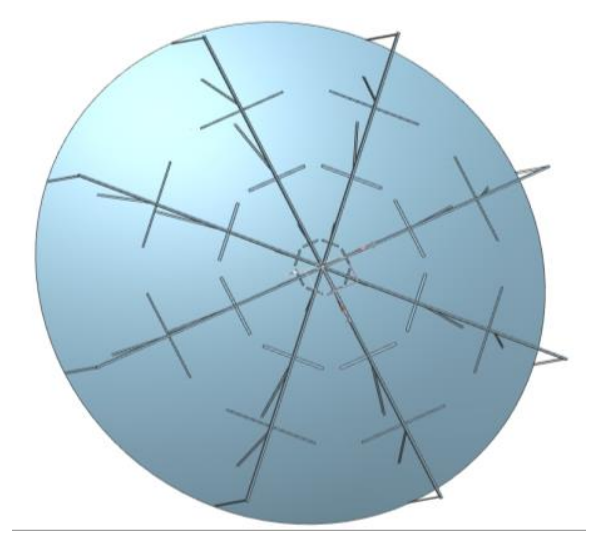

Fig. 1. Geometric model of the transformable antenna reflector.

Finite element models were built based on the geometric model of the transformable antenna reflector. To make the solution results invariant with mesh density, the modeling was done on meshes with different density; the chosen finite element models had from 400000 to 440000 hexagonal and prismatic elements finite elements.

The temperature state of the transformable antenna reflector was modeled using Siemens NX software. Material properties for modeling were as follows:

- Frame (CFPR): thermal conductivity $-56 \mathrm{~W} /(\mathrm{m} \cdot \mathrm{K})$, specific heat $-1000 \mathrm{~J} /(\mathrm{kg} \cdot \mathrm{K})$;

- Mesh sheet (tungsten fibers): thermal conductivity- $3.6 \mathrm{~W} /(\mathrm{m} \cdot \mathrm{K})$, specific heat -500 $\mathrm{J} /(\mathrm{kg} \cdot \mathrm{K})$

- Web (aramid): thermal conductivity $-0.26 \mathrm{~W} /(\mathrm{m} \cdot \mathrm{K})$, specific heat $-800 \mathrm{~J} /(\mathrm{kg} \cdot \mathrm{K})$.

Incident and Earth-reflected solar radiation fluxes, as well as the Earth's thermal radiation flux were defined for the conditions of the flight on a geostationary orbit. The analysis of the temperature state of the transformable antenna reflector was done in the characteristic points of the orbit: illumination by the Sun from the rear side, side illumination by the Sun, entering the Earth shadow and staying in the Earth shadow.

The calculated thermal fluxes were used as the input data for calculating the temperature state. Figure 2 shows the temperature distribution on the transformable antenna reflector for different positions on the orbit.

The maximum temperature of the transformable antenna reflector is $164{ }^{\circ} \mathrm{C}$, and the minimum reflector temperature is $-140{ }^{\circ} \mathrm{C}$.

The stress-strain state was analyzed using ANSYS. The calculation was done for the case of side illumination by the Sun when the maximum temperature gradients take place, leading to high stresses and strains.

It was assumed that the transformable antenna reflector is rigidly fixed in the junction point of the deployable rods. The following assumptions were used for mathematical modeling:

- mesh sheet was treated as a continuous thin-walled shell;

- interaction at the boundary was modeled using contact finite elements;

- the variation of materials' physico-mechanical and thermal properties with temperature was neglected.

Individual results of the calculation of displacement fields in the transformable antenna reflector for the temperature state from the side illumination by the Sun are shown in Fig. 3 and Fig. 4.

Based on the mathematical modeling of the stress-strain state of the transformable antenna reflector, it was established that the maximum displacement is $201 \mathrm{~mm}$, and the maximum normal stress is $0.63 \mathrm{MPa}$. However, due to the reflector construction and the reinforcement from the web system, the deformation of the mesh sheet is less than $38 \mathrm{~mm}$ for the aperture diameter of $100 \mathrm{~m}$, which is less than $0.04 \%$. 


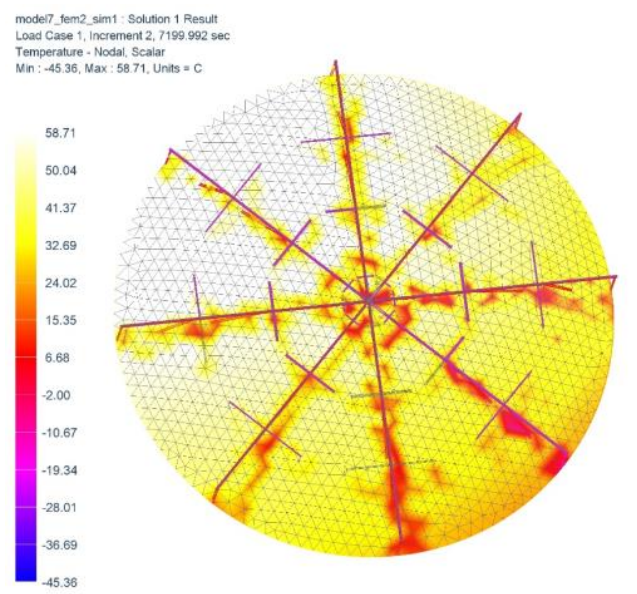

Units $=\mathrm{C}$

a

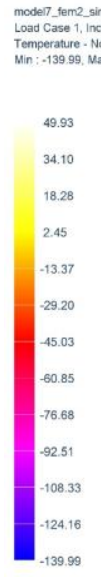

Units $=c$

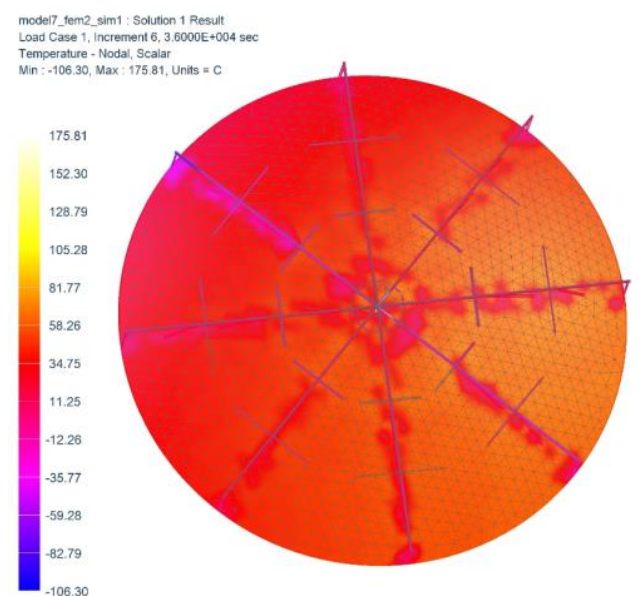

Units $=\mathrm{c}$

b

model7 ferm2_sim1 : Solution 1 Result
Load Case 1 , Increment $12,5.0400 \mathrm{E}+004 \mathrm{sec}$ Load Case 1, Increment $12,5.0400 \mathrm{E}+004 \mathrm{~s}$
Temperature - Nodal, Scalat

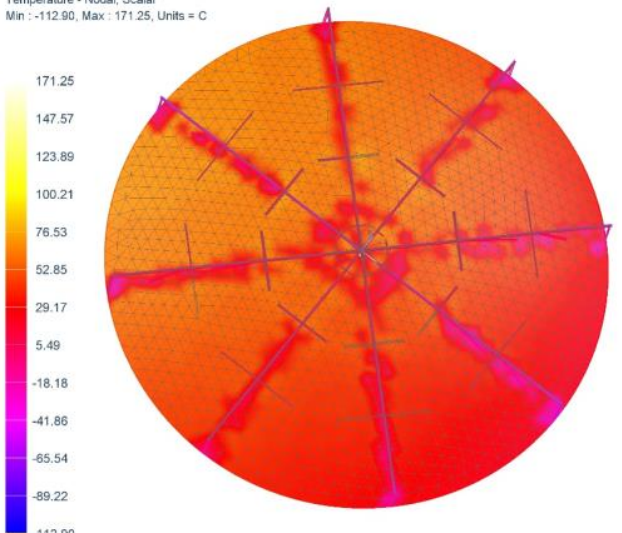

Units $=\mathrm{C}$

c

d

Fig. 2. Temperature state of the transformable antenna reflector on the orbit, ${ }^{\circ} \mathrm{C}: \mathrm{a}-$ illumination by the Sun from the rear side; $b$ - side illumination by the Sun; c - entering the Earth shadow; $\mathrm{d}$ - staying in the Earth shadow.
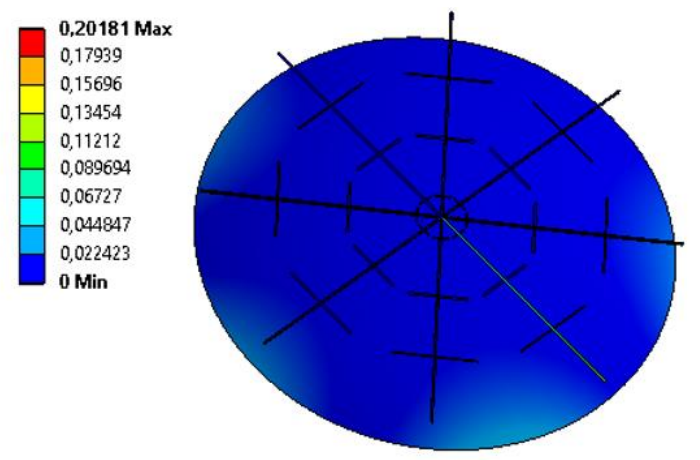

0,00
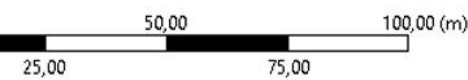

Fig. 3. Total displacements of the transformable antenna reflector, $\mathrm{m}$. 

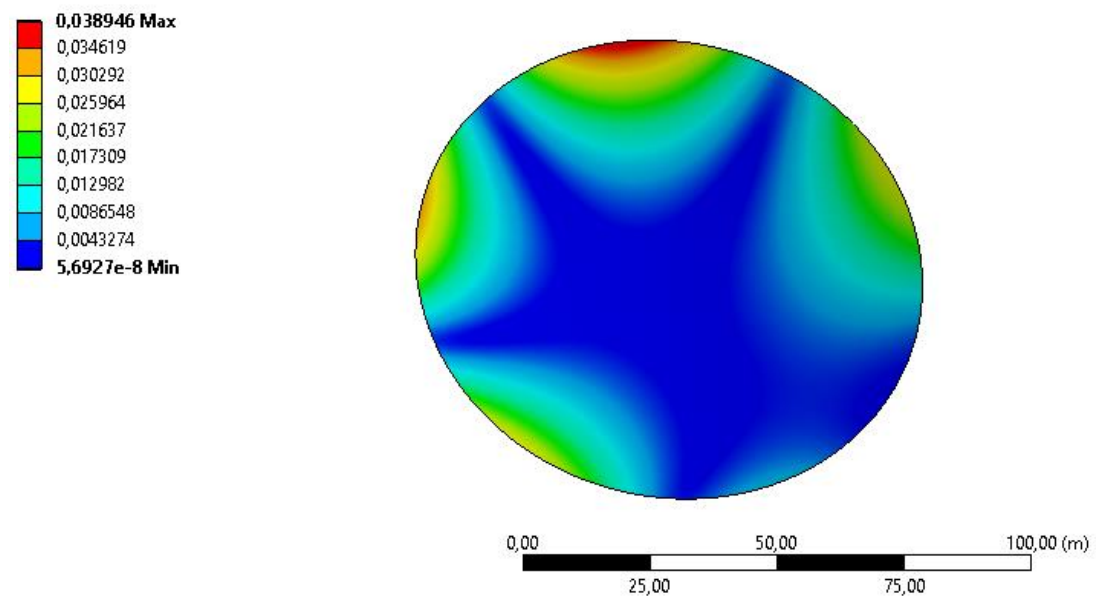

Fig. 4. Total displacements of the mesh sheet of the transformable antenna reflector, $\mathrm{m}$.

\section{Conclusions}

This paper proposed the method of choosing geometric models of transformable antenna reflectors from polymer composite materials using parametric modeling. The method takes into account periodic factors of the operating environment such as ultra-high vacuum, incident and reflected solar radiation, the Earth's thermal radiation, temperature cycling.

This method was used to assess the workability of the transformable antenna reflector structure with a diameter of $100 \mathrm{~m}$ and calculate its temperature and stress-strain state for typical operating conditions on the geostationary orbit.

\section{References}

1. Kotik A., Usyukin V., Vinogradov I. and Arkhipov M. Proc. of SPIE - The Int. Soc. for Optical Engineering, 10567, 105671A, 6 p. DOI: 10.1117/12.2308118 (2017)

2. Reznik S.V., Prosuntsov P.V., Mikhailovsky K.V. and Shafikova I.R. IOP Conference Series: Materials Science and Engineering 153 1. 012012, 10 p. DOI: 10.1088/1757899X/153/1/012001 (2016)

3. Sayapin S.N. and Shkapov P.M. J. of Machinery Manufacture and Reliability, 45. pp. 387-397. (2016)

4. Sayapin S N J. of Machinery Manufacture and Reliability 46, 6 pp. 519-526. (2017)

5. Golovatov D., Mikhaylov M. and Bosov A. J. of Science and Technology 9, 46 No 107492 (2016) DOI: 10.17485/ijst/2016/v9i46/107492

6. Usyukin V.I., Arkhipov Yu.M. Static and dynamic modeling of large space antenna reflectors: handbook (Moscow: Bauman Moscow State Technical University Press, 2015)

7. Smirnov A.V., Baryshev A.M, Pilipenko S.V., et al. Space mission Millimetron for terahertz astronomy // Proceeding of SPIE, 8442, 9 p. DOI: 10.1117/12.927184 21 (2012)

8. Zimin V. N. Development of a method for dynamics analysis and workability assessment of large transformable truss space structures (Moscow: Bauman Moscow State Technical University Press, 2008)

9. Arkhipov M.Yu., Telepnev P.P. Cosmic research 52, 5 pp. 418-422. (2014)

10. Imbriale W. Spaceborne antennas for planetary exploration (N.Y.: John Wiley and Sons, 2006) 